\title{
Does body mass index or waist-hip ratio correlate with arterial stiffness based on brachial-ankle pulse wave velocity in Chinese rural adults with hypertension?
}

Feng Hu, ${ }^{1,2}$, Rihua Yu ${ }^{3}$, Fengyu Han 1,2, Juan Li ${ }^{4}$, Wei Zhou ${ }^{2,5}$, Tao Wang ${ }^{2,5}$, Lingjuan Zhu ${ }^{2,5}$, Xiao Huang ${ }^{1,2,5}$, Huihui Bao ${ }^{1,2,5^{*}}$ and Xiaoshu Cheng ${ }^{1,2,5^{*}}$

\begin{abstract}
Background: The relationship between obesity indices and arterial stiffness (AS) has not been fully discovered nor has it been studied in depth in large hypertensive patient populations. The aim of this study was to explore the association between body mass index (BMI) and waist-hip ratio (WHR) levels and AS based on brachial-ankle pulse wave velocity (baPWV) in Chinese rural adults with hypertension.

Methods: This cross-sectional study analyzed 5049 Chinese rural adults with essential hypertension. BMI was calculated as the body weight in kilograms divided by the square of the height in meters $\left(\mathrm{kg} / \mathrm{m}^{2}\right)$. Central obesity was defined as WHR $\geq 0.9$ for males and $\geq 0.85$ for females. Measurement of arterial stiffness was carried out via brachialankle pulse wave velocity (baPWV).
\end{abstract}

Results: The prevalence of overweight, general obesity, central obesity and increased AS were $26.88 \%, 3.39 \%, 63.85 \%$ and $44.01 \%$, respectively. Multivariate logistic regression analysis indicated that BMI levels were negatively associated with the prevalence of increased AS (adjusted-OR per SD increase: $0.74,95 \% C / 0.67-0.81, P<0.001$ ). When BMI was instead treated as a categorical variable divided into tertiles, the same relationship was observed ( $\mathrm{P}$ for trend $<0.001$ ). Inversely, WHR levels were positively associated with the prevalence of increased AS (adjusted-OR per SD increase: $1.25,95 \% \mathrm{Cl} 1.14-1.36, \mathrm{P}<0.001$ ). Compared to subjects without central obesity, those with central obesity had a higher prevalence of increased AS (adjusted-OR: 1.52, 95\% Cl 1.28-1.81, $\mathrm{P}<0.001$ ). Linear regression models indicated similar results in the correlation between BMI or WHR levels and baPWV levels (adjusted- $\beta$ per SD increase: $-0.57,95 \%$ $C l-0.68$ to $-0.46, P<0.001$; adjusted $\beta$ per SD increase: $4.46,95 \% C / 3.04-5.88, P<0.001$ ). There were no interactions in terms of age and blood pressure on the relationship between BMI or WHR levels and the prevalence of increased AS or baPWV levels.

Conclusion: There was an inverse relationship between BMI levels and increased AS or baPWV levels, whereas WHR levels and central obesity were positively associated with increased AS or baPWV levels in Chinese rural adults with hypertension.

*Correspondence: huihui_bao77@126.com; xiaoshumenfan126@163.com

1 Department of Cardiovascular Medicine, The Second Affiliated Hospital

of Nanchang University, No. 1 Minde Road, Nanchang 330006, Jiangxi,

China

Full list of author information is available at the end of the article

(c) The Author(s) 2021. Open Access This article is licensed under a Creative Commons Attribution 4.0 International License, which permits use, sharing, adaptation, distribution and reproduction in any medium or format, as long as you give appropriate credit to the original author(s) and the source, provide a link to the Creative Commons licence, and indicate if changes were made. The images or other third party material in this article are included in the article's Creative Commons licence, unless indicated otherwise in a credit line to the material. If material is not included in the article's Creative Commons licence and your intended use is not permitted by statutory regulation or exceeds the permitted use, you will need to obtain permission directly from the copyright holder. To view a copy of this licence, visit http://creativecommons.org/licenses/by/4.0/. The Creative Commons Public Domain Dedication waiver (http://creativeco mmons.org/publicdomain/zero/1.0/) applies to the data made available in this article, unless otherwise stated in a credit line to the data. 
Keywords: Body mass index, Waist-hip ratio, Arterial stiffness, Brachial-ankle pulse wave velocity, Chinese, Hypertension

\section{Background}

Cardiovascular diseases (CVDs) constitute the leading cause of global mortality including China [1]. The prevalence of global obesity has steadily increased in the past few decades and has increased the risk of CVDs [2]. Approximately $23.2 \%$ of adults in China are estimated to suffer from hypertension, and hypertension is often accompanied by obesity [3]. Arterial stiffness (AS) has a crucial role in the pathogenesis of hypertension, which could prognosticate the risk rate of subclinical vascular disorders and cardiovascular events in hypertensive patients [4]. Increased AS represents a valuable metric to guide the stratification of hypertensive patients based on their cardiovascular risk [5].

Carotid-femoral pulse wave velocity (cfPWV) is currently considered as the gold standard methodology for evaluating AS [6], whereas brachial-ankle pulse wave velocity (baPWV) is equally regard as a risk biomarker of CVDs and is strongly linked to aortic PWV and cfPWV [7]. In the same way, baPWV has been accepted as an effective and repeatable metric tool to assessing AS in many epidemiological surveys investigation [8-13]. Therefore, baPWV was adopted as a metric for AS in this study.

Body mass index (BMI) is one of the main measures for general obesity and is commonly used in clinical practice. However, research on the association between $\mathrm{BMI}$ and PWV in various populations, including hypertensive patients [14-19], remains controversial. Studies have reported an insignificant relationship [14, 18, 20 24], positive relationship [15, 17, 19, 25-28], or negative relationship [16, 29-33]. Many causes could interpret these apparent discrepancies including diverse sample size, different origins of the study population, ethnic and regional disparity. On the other hand, BMI is not able to differentiate fat-free muscle from fat mass. Furthermore, BMI cannot account for sex and racial differences in fat content, and it is not able to differentiate the distribution of visceral and subcutaneous fat [34]. Thus, more studies need to verify the relationship between the BMI levels and AS based on PWV.

Waist-hip ratio (WHR), a metric of central obesity less influenced by muscle and bone mass, might be better indicate risk for CVDs associated with obesity than BMI $[35,36]$. Higher WHR was significantly correlated with increased AS based on PWV [19, 25, 27]. This study was designed for evaluating the relationship between the BMI or WHR levels and the prevalence of increased AS and baPWV in a large real-world population of Chinese hypertensive rural adults.

\section{Methods \\ Participants}

All subjects in the present study were participants in the ongoing China H-type hypertension Registry Study (Registration number: ChiCTR1800017274).The data collection approaches and the established standards of inclusion or exclusion have been described previously $[37,38]$. Briefly, the study is a real-world, multicenter, observational study, conducted in March 2018 at Wuyuan, Jiangxi province of China. Enrolled population were hypertensive patients who aged 18 years and older. The exclusion criteria included psychological or nervous system impairment resulting in an inability to demonstrate informed consent, unable to be long-term followed-up according to the study protocol, or plans to relocate in the near future.

Of the eligible patients, 5, 233 completed baPWV measurements. After excluding 2 participants without BMI measurements, 78 individuals with ankle brachial index $(\mathrm{ABI})<0.9[11,24]$ and 104 patients with atrial fibrillation, finally 5, 049 participants were included in our analysis (Additional file 2: Fig. 1).

\section{Clinical data collection}

Participants' demographic characteristics (age, gender), lifestyle factors (smoking and drinking status), medical history [diabetes mellitus (DM), coronary heart disease (CHD), stroke, dyslipidemia and atrial fibrillation] and medication usage were gathered by professional researchers through questionnaire survey. Atrial fibrillation was diagnosed based on a medical history and through resting supine standard 12-lead surface electrocardiogram.

Anthropometric measurements for each patient including weight, height, waistline, hipline, systolic blood pressure (SBP), diastolic blood pressure (DBP) and heart rate were obtained by researchers. Waistline and hipline were measured using an inelastic measuring tape Blood pressure (BP) was measured, with the participant in a sitting position using the Omron HBP-1300 Professional Portable Blood Pressure Monitor (Kyoto, Japan) on the right arm, which was supported at the heart level. After a 5-min rest period, BP was measured four times, and SBP and DBP were calculated as the average of the last three readings. BMI was calculated as the body weight in kilograms divided by the square of the height in meters 
$\left(\mathrm{kg} / \mathrm{m}^{2}\right)$. WHR was calculated as the waistline in centimeters divided by the hipline in centimeters. In our study, subjects were divided into three categories according to BMI levels $\left(<25 \mathrm{~kg} / \mathrm{m}^{\wedge}\right.$, control group; $25-30 \mathrm{~kg} / \mathrm{m}^{\wedge}$, overweight; and $\geq 30 \mathrm{~kg} / \mathrm{m}^{\wedge}$, general obesity) [25-27, 30]. Central obesity was defined as WHR $\geq 0.9$ for males and $\geq 0.85$ for females [39].

\section{Laboratory assay}

Blood samples were collected utilizing venipuncture after an overnight fast of at least $12 \mathrm{~h}$. The levels of plasma total homocysteine, fasting blood glucose (FBG), total cholesterol (TC), total triglyceride (TG), high-density lipoprotein cholesterol (HDL-C), low-density lipoprotein cholesterol (LDL-C), serum uric acid and creatinine, blood urea nitrogen (BUN), total and direct bilirubin, aspartate aminotransferase (AST) and alanine aminotransferase (ALT) were measured using automatic clinical analyzers (Beckman Coulter) In our analysis, diagnosis of incident diabetes was defined as fasting glucose $>7.0 \mathrm{mmol} / \mathrm{l}$, and/or self-reported diabetes.. Dyslipidemia was defined as having one or more of the following features: elevated TG $(\geq 2.3 \mathrm{mmol} / \mathrm{L})$, elevated $\mathrm{TC}(\geq 6.2 \mathrm{mmol} / \mathrm{L})$, elevated LDL-C $(\geq 4.1 \mathrm{mmol} / \mathrm{L})$ and reduced HDL-C $(<1.0 \mathrm{mmol} / \mathrm{L})$ or on appropriate lipidlowering medication..The estimated glomerular filtration rate (eGFR) was calculated by the equation of Chronic Kidney Disease Epidemiology Collaboration (CKD-EPI).

\section{Measurement of baPWV and definition of arterial stiffness} BaPWV and the ABI were measured using a BP203RPEIII networked arteriosclerosis detection device (Omron Health Care, Kyoto, Japan). Participants received this measurement after at least 5 min of rest in the supine position in a quiet room. The measurement approaches of baPWV and ABI related to this study have been described previously [37, 38]. The average of baPWV measured on bilateral limbs of each patient was used for analysis. While baPWV value has been shown to have prognostic significance, with a threshold of increased risk that exist around $18 \mathrm{~m} / \mathrm{s}$ [11]. Therefore, baPWV $\geq 18 \mathrm{~m} / \mathrm{s}$ was considered as increased AS in our analysis.

\section{Statistical analysis}

Continuous variables are presented as the means \pm standard deviation (SD) or the median (quartiles), as appropriate, and are compared using the Student's t test, one-way analysis or the Mann Whitney $U$ test, depending on whether the quantitative data were consistent with a normal distribution. Categorical variables were presented as count (percentage), differences between groups were measured by chi-square test.
Multivariate logistic regression models based on odds ratio (OR) with their associated 95\% confidence interval (CI) were used to estimate the association of BMI or WHR levels with increased AS. Linear regression models indicated were used to estimate the effect size $(\beta)$ of BMI or WHR levels on baPWV levels. The crude model was not adjusted for any confounder. The model I was adjusted for age, gender, SBP, DBP, heart rate, WHR or BMI. The model II was confounder model. The confounder model screened covariates including age, sex, SBP, DBP, heart rate, BMI, WHR, DM, CHD, history of stroke, smoking and drinking status, total homocysteine, TC, TG, HDL-C, LDL-C, AST, ALT, serum uric acid, eGFR, total and direct bilirubin, antihypertensive medications, lipid-lowering agents and antiplatelet agents except the independent variable itself. We selected these confounders on the basis that, when added to this model, it changed the matched odds ratio by at least 10 percent. Additional file 1: Table 1 showed the association of each confounder with the outcomes of interest. We considered the confounder model to be the main model. In addition, we performed tests for linear trend by entering the median value of each category of BMI or WHR levels as a continuous variable in the models.

Furthermore, the generalized additive model and smooth curve fitting (penalized spline method) was used to visually show the relationship of BMI or WHR levels with baPWV levels. Subgroup analyses were conducted with a stratified multivariate regression approach, and interaction analyses were presented in tabulated form.

All statistical analyses were performed using the statistical package R (http://www.R-project.org, The R Foundation, version 3.4.3) and the Empower (R; www.empow erstats.com; X\&Y Solutions, Inc, Boston, MA, USA). All $\mathrm{P}$-values are two-tailed, and $\mathrm{P}<0.05$ was considered statistically significant.

\section{Results \\ Clinical characteristics of study population}

The present study included 5, 049 Chinese adult hypertensive individuals (mean age: $64.46 \pm 9.45$ years, range 29-93 years; male, 49.89\%), and the prevalence of overweight, general obesity, central obesity and increased AS were $26.88 \%, 3.39 \%, 63.85 \%$ and $44.01 \%$, respectively. The clinical characteristics of the study participants grouped by BMI tertiles or central obesity were presented in Table 1. Compared to $\mathrm{BMI} \leq 21.68 \mathrm{~kg} / \mathrm{m}^{\wedge 2}$, there were lower age and SBP, smaller baPWV levels s, a lower prevalence of increased AS, greater DBP and WHR, a higher prevalence of central obesity for the participants in the second and highest tertile of BMI (all $P$ values $<0.05$, Table 1). Compared to subjects without central obesity, those with central obesity had lower age, greater BMI and 
Table 1 Clinical characteristics of participants grouped by BMI tertiles or central obesity

\begin{tabular}{|c|c|c|c|c|c|c|c|}
\hline \multirow[t]{2}{*}{ Characteristics } & \multicolumn{3}{|c|}{ BMI tertiles $\left(\mathrm{kg} / \mathrm{m}^{\wedge^{2}}\right)$} & \multirow[t]{2}{*}{ P-value } & \multicolumn{2}{|l|}{ Central obesity } & \multirow[t]{2}{*}{$P$-value } \\
\hline & $\mathrm{T} 1[13.83,21.68]$ & T2 [21.68, 24.69] & T3 $[24.69,46.43]$ & & No & Yes & \\
\hline Number of subjects (n) & 1683 & 1683 & 1683 & & 1825 & 3224 & \\
\hline Age (years) & $68.13 \pm 8.84$ & $63.89 \pm 8.72$ & $61.35 \pm 9.51$ & $<0.001$ & $64.98 \pm 9.61$ & $64.16 \pm 9.35$ & 0.004 \\
\hline Male, n (\%) & 898 (53.36\%) & $820(48.72 \%)$ & 801 (47.59\%) & 0.002 & $1183(64.82 \%)$ & $1336(41.44 \%)$ & $<0.001$ \\
\hline $\mathrm{SBP}(\mathrm{mmHg})$ & $147.81 \pm 18.37$ & $147.00 \pm 17.72$ & $146.13 \pm 16.49$ & 0.021 & $147.05 \pm 18.26$ & $146.94 \pm 17.15$ & 0.845 \\
\hline $\mathrm{DBP}(\mathrm{mmHg})$ & $86.30 \pm 11.24$ & $88.99 \pm 10.51$ & $90.82 \pm 10.55$ & $<0.001$ & $88.24 \pm 11.14$ & $88.97 \pm 10.80$ & 0.054 \\
\hline HR (times/min) & $75.40 \pm 15.56$ & $74.98 \pm 13.50$ & $76.89 \pm 13.91$ & $<0.001$ & $74.16 \pm 14.44$ & $76.66 \pm 14.26$ & $<0.001$ \\
\hline Height (cm) & $155.22 \pm 8.11$ & $155.90 \pm 8.09$ & $156.51 \pm 8.27$ & $<0.001$ & $157.35 \pm 7.72$ & $155.05 \pm 8.30$ & $<0.001$ \\
\hline Weigh (kg) & $47.41 \pm 6.25$ & $56.58 \pm 6.30$ & $66.62 \pm 8.60$ & $<0.001$ & $52.29 \pm 8.85$ & $59.46 \pm 10.64$ & $<0.001$ \\
\hline $\mathrm{BMI}\left(\mathrm{kg} / \mathrm{m}^{\wedge}{ }^{2}\right)$ & $19.62 \pm 1.53$ & $23.21 \pm 0.86$ & $27.14 \pm 2.27$ & $<0.001$ & $21.04 \pm 2.70$ & $24.61 \pm 3.21$ & $<0.001$ \\
\hline BMl category $\left(\mathrm{kg} / \mathrm{m} \wedge^{2}\right)$ & & & & $<0.001$ & & & $<0.001$ \\
\hline Control $(<25)$ & $1683(100.00 \%)$ & $1683(100.00 \%)$ & 155 (9.21\%) & & 1684 (92.27\%) & 1837 (56.98\%) & \\
\hline Overweight $(\geq 25,<30)$ & $0(0.00 \%)$ & $0(0.00 \%)$ & $1357(80.63 \%)$ & & 135 (7.40\%) & $1222(37.90 \%)$ & \\
\hline General obesity $(\geq 30)$ & $0(0.00 \%)$ & $0(0.00 \%)$ & $171(10.16 \%)$ & & $6(0.33 \%)$ & $165(5.12 \%)$ & \\
\hline Waistline $(\mathrm{cm})$ & $73.29 \pm 6.38$ & $82.39 \pm 5.46$ & $91.27 \pm 6.47$ & $<0.001$ & & & \\
\hline Hipline (cm) & $86.03 \pm 4.41$ & $91.39 \pm 4.54$ & $97.05 \pm 5.94$ & $<0.001$ & $89.28 \pm 6.46$ & $92.74 \pm 6.57$ & $<0.001$ \\
\hline WHR & $0.85 \pm 0.06$ & $0.90 \pm 0.06$ & $0.94 \pm 0.06$ & $<0.001$ & $0.83 \pm 0.04$ & $0.94 \pm 0.05$ & $<0.001$ \\
\hline Central obesity, n (\%) & $565(33.57 \%)$ & $1143(67.91 \%)$ & $1516(90.08 \%)$ & $<0.001$ & & & \\
\hline baPWV (m/s) & $18.97 \pm 4.34$ & $17.92 \pm 3.63$ & $17.35 \pm 3.42$ & $<0.001$ & $17.94 \pm 3.85$ & $18.16 \pm 3.89$ & 0.010 \\
\hline Increased AS, n (\%) & $877(52.11 \%)$ & $715(42.48 \%)$ & $630(37.43 \%)$ & $<0.001$ & $743(40.71 \%)$ & $1479(45.87 \%)$ & $<0.001$ \\
\hline Smoking status, n (\%) & & & & $<0.001$ & & & $<0.001$ \\
\hline Never & $771(45.81 \%)$ & $930(55.26 \%)$ & $990(58.82 \%)$ & & 766 (41.97\%) & 1925 (59.71\%) & \\
\hline Former smoker & $292(17.35 \%)$ & $317(18.84 \%)$ & $323(19.19 \%)$ & & $373(20.44 \%)$ & $559(17.34 \%)$ & \\
\hline Current smoker & $620(36.84 \%)$ & 436 (25.91\%) & $370(21.98 \%)$ & & $686(37.59 \%)$ & $740(22.95 \%)$ & \\
\hline Drinking status, n (\%) & & & & 0.076 & & & $<0.001$ \\
\hline Never & $1030(61.20 \%)$ & 1060 (62.98\%) & $1104(65.60 \%)$ & & $1043(57.15 \%)$ & $2151(66.72 \%)$ & \\
\hline Former drinker & $219(13.01 \%)$ & $211(12.54 \%)$ & $179(10.64 \%)$ & & $259(14.19 \%)$ & $350(10.86 \%)$ & \\
\hline Current drinker & $434(25.79 \%)$ & $412(24.48 \%)$ & $400(23.77 \%)$ & & $523(28.66 \%)$ & $723(22.43 \%)$ & \\
\hline Homocysteine ( $\mu \mathrm{mol} / \mathrm{L})$ & 15.75 (12.91-20.88) & 14.87 (12.40-19.20) & $14.93(12.46-18.76)$ & $<0.001$ & $15.62(12.79-21.14)$ & 14.91 (12.50-19.02) & $<0.001$ \\
\hline $\mathrm{FBG}(\mathrm{mmol} / \mathrm{L})$ & $5.80 \pm 1.11$ & $6.18 \pm 1.63$ & $6.44 \pm 1.97$ & $<0.001$ & $5.80 \pm 1.10$ & $6.33 \pm 1.84$ & $<0.001$ \\
\hline $\mathrm{TC}(\mathrm{mmol} / \mathrm{L})$ & $4.99 \pm 1.07$ & $5.13 \pm 1.14$ & $5.25 \pm 1.12$ & $<0.001$ & $4.96 \pm 1.07$ & $5.22 \pm 1.13$ & $<0.001$ \\
\hline $\mathrm{TG}(\mathrm{mmol} / \mathrm{L})$ & $1.28 \pm 0.71$ & $1.81 \pm 1.28$ & $2.18 \pm 1.52$ & $<0.001$ & $1.12(0.83-1.56)$ & $1.63(1.16-2.35)$ & $<0.001$ \\
\hline $\mathrm{HDL}-\mathrm{C}(\mathrm{mmol} / \mathrm{L})$ & $1.63 \pm 0.43$ & $1.47 \pm 0.38$ & $1.38 \pm 0.34$ & $<0.001$ & $1.60 \pm 0.43$ & $1.43 \pm 0.36$ & $<0.001$ \\
\hline $\mathrm{LDL}-\mathrm{C}(\mathrm{mmol} / \mathrm{L})$ & $2.71 \pm 0.74$ & $2.94 \pm 0.80$ & $3.12 \pm 0.79$ & $<0.001$ & $2.71 \pm 0.74$ & $3.04 \pm 0.80$ & $<0.001$ \\
\hline $\begin{array}{l}\text { Serum uric acid } \\
(\mathrm{mmol} / \mathrm{L})\end{array}$ & $414.96 \pm 117.95$ & $425.37 \pm 117.41$ & $454.13 \pm 125.18$ & $<0.001$ & $422.65 \pm 116.69$ & $436.49 \pm 123.63$ & $<0.001$ \\
\hline $\begin{array}{l}\text { Serum creatinine } \\
(\mathrm{mmol} / \mathrm{L})\end{array}$ & $68.00(57.00-84.00)$ & $67.00(55.00-81.00)$ & $68.00(57.00-84.00)$ & 0.046 & $71.00(59.00-86.00)$ & $66.00(55.00-81.00)$ & $<0.001$ \\
\hline BUN (mmol/L) & $5.65 \pm 2.07$ & $5.37 \pm 1.69$ & $5.30 \pm 1.70$ & $<0.001$ & $5.58 \pm 2.05$ & $5.36 \pm 1.70$ & 0.002 \\
\hline eGFR $\left(\mathrm{ml} / \mathrm{min} / 1.73 \mathrm{~m}^{2}\right)$ & $83.19 \pm 19.96$ & $87.36 \pm 18.69$ & $87.62 \pm 19.87$ & $<0.001$ & $85.33 \pm 20.32$ & $86.47 \pm 19.19$ & 0.120 \\
\hline Total bilirubin (mmol/L) & $14.21 \pm 6.59$ & $14.23 \pm 6.06$ & $14.62 \pm 6.35$ & 0.107 & $14.42 \pm 6.24$ & $14.31 \pm 6.40$ & 0.306 \\
\hline Direct bilirubin (mmol/L) & $5.45 \pm 2.23$ & $5.28 \pm 1.96$ & $5.35 \pm 1.95$ & 0.269 & $5.54 \pm 2.13$ & $5.26 \pm 2.00$ & $<0.001$ \\
\hline AST $(U / L)$ & $24.00(20.00-30.00)$ & $24.00(20.00-29.00)$ & $24.00(20.00-31.00)$ & 0.003 & $24.00(20.00-30.00)$ & $24.00(20.00-30.00)$ & 0.212 \\
\hline ALT (U/L) & $15.00(11.00-19.00)$ & $17.00(13.00-23.00)$ & $21.00(15.00-29.00)$ & $<0.001$ & $15.00(12.00-21.00)$ & $18.00(13.00-26.00)$ & $<0.001$ \\
\hline $\mathrm{DM}, \mathrm{n}(\%)$ & $199(11.82 \%)$ & $323(19.19 \%)$ & $413(24.54 \%)$ & $<0.001$ & 199 (10.90\%) & $736(22.83 \%)$ & $<0.001$ \\
\hline CHD, n (\%) & $116(6.89 \%)$ & $104(6.18 \%)$ & $114(6.77 \%)$ & 0.672 & $111(6.08 \%)$ & $223(6.92 \%)$ & \\
\hline History of stroke, n (\%) & $130(7.72 \%)$ & $132(7.84 \%)$ & $113(6.71 \%)$ & 0.390 & 137 (7.51\%) & $238(7.38 \%)$ & \\
\hline Dyslipidemia, n (\%) & $364(21.63 \%)$ & $669(39.75 \%)$ & $818(48.60 \%)$ & $<0.001$ & $440(24.11 \%)$ & $1411(43.77 \%)$ & $<0.001$ \\
\hline $\begin{array}{l}\text { Antihypertensive medi- } \\
\text { cations, n (\%) }\end{array}$ & $938(55.73 \%)$ & $1056(62.75 \%)$ & $1084(64.41 \%)$ & $<0.001$ & 1037 (56.82\%) & $2041(63.31 \%)$ & $<0.001$ \\
\hline
\end{tabular}


Table 1 (continued)

\begin{tabular}{|c|c|c|c|c|c|c|c|}
\hline \multirow[t]{2}{*}{ Characteristics } & \multicolumn{3}{|c|}{ BMI tertiles $\left(\mathrm{kg} / \mathrm{m}^{\wedge^{2}}\right)$} & \multirow[t]{2}{*}{$P$-value } & \multicolumn{2}{|c|}{ Central obesity } & \multirow[t]{2}{*}{$P$-value } \\
\hline & T1 [13.83, 21.68] & T2 $[21.68,24.69]$ & T3 $[24.69,46.43]$ & & No & Yes & \\
\hline $\begin{array}{l}\text { Hypoglycemic agents, } \\
\text { n (\%) }\end{array}$ & $39(2.32 \%)$ & 84 (4.99\%) & $101(6.00 \%)$ & $<0.001$ & $30(1.64 \%)$ & $194(6.02 \%)$ & $<0.001$ \\
\hline $\begin{array}{l}\text { Lipid-lowering agents, } \\
\mathrm{n}(\%)\end{array}$ & $33(1.96 \%)$ & $62(3.68 \%)$ & 77 (4.58\%) & $<0.001$ & 47 (2.58\%) & $125(3.88 \%)$ & 0.014 \\
\hline $\begin{array}{l}\text { Antiplatelet agents, } \\
\mathrm{n}(\%)\end{array}$ & $43(2.55 \%)$ & 65 (3.86\%) & $76(4.52 \%)$ & 0.008 & $58(3.18 \%)$ & $126(3.91 \%)$ & 0.184 \\
\hline
\end{tabular}

BMI, body mass index; SBP, systolic blood pressure; DBP, diastolic blood pressure; HR, heart rate; WHR, waist hip rate; baPWV, brachial-ankle pulse wave velocity; AS, arterial stiffness; FBG, fasting blood glucose; TC, total cholesterol; TG, total triglyceride; HDL-C, high-density lipoprotein cholesterol; LDL-C, low-density lipoprotein cholesterol; BUN, blood urea nitrogen; eGFR, estimated glomerular filtration rate; AST, aspartate aminotransferase; ALT, alanine aminotransferase; DM, diabetes mellitus; CHD, coronary heart disease

baPWV levels, a higher prevalence of increased AS and overweight as well as general obesity (all $P$ values $<0.05$, Table 1). In this study, 184 patients with diabetes, CHD or stroke were treated with antiplatelet drugs and thus, further adjustments for these conditions were performed in different regression models.

There was a significantly positive correlation between the BMI levels and the WHR levels $(r=0.55, P<0.001$, Additional file 3: Fig. 2). Linear regression models indicated that BMI levels were positively associated with WHR levels ( $\beta$ per $S D$ increase: 0.04, 95\% CI 0.04-0.04, $P<0.001$; Additional file 1: Table 2). Multivariate logistic regression analysis suggested that BMI levels were positively associated with the prevalence of central obesity (adjusted-OR per $S D$ increase: 4.41, 95\% CI 4.024.84, $P<0.001$; Additional file 1: Table 2). Compared to $\mathrm{BMI} \leq 21.68 \mathrm{~kg} / \mathrm{m}^{\wedge}$, there were greater WHR values and a higher prevalence of central obesity for the participants in the second and highest tertile of BMI $(\beta: 0.05$, 95\% CI 0.05-0.05, $P<0.001 ; \beta$ : 0.09, 95\% CI 0.09-0.09, $P<0.001$, respectively; $P$ for trend $<0.001$. OR: $4.19,95 \%$ CI 3.63-4.84, $P<0.001$; OR: 17.96, 95\% CI 14.87-21.70, $P<0.001$, respectively; $P$ for trend $<0.001$; Additional file 1: Table 2).

Clinical characteristics of participants grouped by baPWV quartiles were also presented in Additional file 1: Table 3. Compared to baPWV $\leq 15.36 \mathrm{~m} / \mathrm{s}$, there were reduced eGFR and BMI levels, a lower prevalence of overweight or general obesity, greater extents of age, SBP and DBP, an higher prevalence of central obesity in the third and highest baPWV quartiles (all $P$ values $<0.05$, Additional file 1: Table 3).

\section{Association between BMI levels and the prevalence of increased arterial stiffness}

Multivariate logistic regression analysis indicated that BMI levels were negatively associated with the prevalence of increased AS (adjusted-OR per SD increase: 0.74,
95\% CI 0.67-0.81, $P<0.001$; Table 2). Compared to control group, patients with overweight and general obesity had a lower prevalence of increased AS (adjusted-OR: 0.73, 95\% CI 0.61-0.87, $P<0.001$; adjusted-OR: 0.51, 95\% CI 0.34-0.78, $P=0.002$, respectively; $P$ for trend $<0.001$; Table 2). Compared to $\mathrm{BMI} \leq 21.68 \mathrm{~kg} / \mathrm{m}^{\wedge}$, there were a lower prevalence of increased AS for the participants in the second and highest tertile of BMI (adjusted-OR: 0.71, 95\% CI 0.59-0.86, $P<0.001$; adjusted-OR: 0.56, 95\% $C I$ 0.45-0.69, $P<0.001$, respectively; $P$ for trend $<0.001$; Table 2).

In consideration of that increased AS is closely related to age and BP [11-13, 32, 40, 41], stratified and interaction analyses we ceronducted to explore the effects of age, SBP, and DBP on the association between BMI levels and the prevalence of increased AS. Subgroup analyses indicated that the inverse relationship between BMI levels and the prevalence of increased AS was still stable in the tertiles of age stratification no matter what using BMI levels as continuous variable or the lower tertile of BMI as reference variable $(P$-value for interaction was 0.770 in the main confounder model, Additional file 1: Table 4). Likewise, subgroup analyses also indicated that the reverse relationship between BMI levels and the prevalence of increased AS was still stable in the tertiles of SBP or DBP stratification no matter what using BMI levels as continuous variable or the lower tertile of BMI as reference variable $(P$-value for interaction respectively were 0.566 and 352 in the main confounder model, Additional file 1: Table 5 and 6).

\section{Association between WHR levels and the prevalence of increased arterial stiffness}

Multivariate logistic regression analysis indicated that WHR levels were positively associated with the prevalence of increased AS (adjusted-OR per SD increase: 1.25, 95\% CI 1.14-1.36, $P<0.001$; Table 3). Compared to subjects without central obesity, those with central obesity 
Table 2 Relationship between BMI levels and the prevalence of increased arterial stiffness in different models

\begin{tabular}{|c|c|c|c|c|c|c|c|}
\hline \multirow[t]{2}{*}{ Variables } & \multirow[t]{2}{*}{ Event, n (\%) } & \multicolumn{2}{|l|}{ Crude Model } & \multicolumn{2}{|l|}{ Model I } & \multicolumn{2}{|l|}{ Model II } \\
\hline & & OR $(95 \% \mathrm{Cl})$ & $P$-value & OR $(95 \% \mathrm{Cl})$ & P-value & OR $(95 \% \mathrm{Cl})$ & $P$-value \\
\hline \multicolumn{8}{|l|}{$\mathrm{BMI}\left(\mathrm{kg} / \mathrm{m} \wedge^{2}\right)$} \\
\hline Per SD increase & $2222(44.01 \%)$ & $0.77(0.73,0.82)$ & $<0.001$ & $0.80(0.74,0.88)$ & $<0.001$ & $0.74(0.67,0.81)$ & $<0.001$ \\
\hline \multicolumn{8}{|l|}{ BMl category $\left(\mathrm{kg} / \mathrm{m}^{\wedge^{2}}\right)$} \\
\hline Control $(<25)$ & $1653(46.95 \%)$ & Ref & & Ref & & Ref & \\
\hline Overweight $(\geq 25,<30)$ & $514(37.88 \%)$ & $0.69(0.61,0.78)$ & $<0.001$ & $0.80(0.67,0.95)$ & $<0.001$ & $0.73(0.61,0.87)$ & $<0.001$ \\
\hline General obesity $(\geq 30)$ & $55(32.16 \%)$ & $0.54(0.39,0.74)$ & $<0.001$ & $0.56(0.37,0.84)$ & 0.005 & $0.51(0.34,0.78)$ & 0.002 \\
\hline P-value for trend & & $<0.001$ & & $<0.001$ & & $<0.001$ & \\
\hline \multicolumn{8}{|l|}{ BMI tertiles $\left(\mathrm{kg} / \mathrm{m}^{\wedge}{ }^{2}\right)$} \\
\hline T1 $[13.83,21.68]$ & $877(52.11 \%)$ & Ref & & $\operatorname{Ref}$ & & Ref & \\
\hline T2 $[21.68,24.69]$ & $715(42.48 \%)$ & $0.68(0.59,0.78)$ & $<0.001$ & $0.81(0.67,0.96)$ & 0.018 & $0.71(0.59,0.86)$ & $<0.001$ \\
\hline T3 $[24.69,46.43]$ & $630(37.43 \%)$ & $0.55(0.48,0.63)$ & $<0.001$ & $0.67(0.54,0.82)$ & $<0.001$ & $0.56(0.45,0.69)$ & $<0.001$ \\
\hline$P$-value for trend & & $<0.001$ & & $<0.001$ & & $<0.001$ & \\
\hline
\end{tabular}

$\mathrm{BMI}$, body mass index; Ref, reference; OR, odds ratio; $\mathrm{Cl}$, confidence interval; $S D$, standard deviation

Model I adjusted for age, sex, SBP, DBP, HR and WHR

Model Il adjusted for age, sex, SBP, DBP, HR, WHR, smoking status, TG, LDL-C, eGFR, DM, CHD, history of stroke and antihypertensive medications

Table 3 Relationship between WHR levels and the prevalence of increased arterial stiffness

\begin{tabular}{|c|c|c|c|c|c|c|c|}
\hline \multirow[t]{2}{*}{ Variables } & \multirow[t]{2}{*}{ Event, n (\%) } & \multicolumn{2}{|l|}{ Crude Model } & \multicolumn{2}{|l|}{ Model I } & \multicolumn{2}{|l|}{ Model II } \\
\hline & & OR $(95 \% \mathrm{Cl})$ & $P$-value & OR $(95 \% \mathrm{Cl})$ & $P$-value & OR $(95 \% \mathrm{Cl})$ & $P$-value \\
\hline \multicolumn{8}{|l|}{ WHR } \\
\hline Per SD increase & 2222 (44.01\%) & $1.11(1.05,1.18)$ & $<0.001$ & $1.28(1.18,1.40)$ & $<0.001$ & $1.25(1.14,1.36)$ & $<0.001$ \\
\hline \multicolumn{8}{|l|}{ Central obesity } \\
\hline No & $743(40.71 \%)$ & Ref & & Ref & & Ref & \\
\hline Yes & 1479 (45.87\%) & $1.23(1.10,1.39)$ & $<0.001$ & $1.62(1.36,1.92)$ & $<0.001$ & $1.52(1.28,1.81)$ & $<0.001$ \\
\hline \multicolumn{8}{|l|}{ WHR tertiles } \\
\hline $\mathrm{T} 1[0.53,0.87]$ & 697 (41.41\%) & Ref & & Ref & & Ref & \\
\hline T2 $[0.87,0.93]$ & 716 (42.70\%) & $1.05(0.92,1.21)$ & 0.452 & $1.41(1.18,1.69)$ & $<0.001$ & $1.36(1.14,1.64)$ & $<0.001$ \\
\hline T3 $[0.93,1.68]$ & 809 (47.90\%) & $1.30(1.14,1.49)$ & $<0.001$ & $1.93(1.57,2.36)$ & $<0.001$ & $1.81(1.47,2.22)$ & $<0.001$ \\
\hline$P$ for trend & & $<0.001$ & & $<0.001$ & & $<0.001$ & \\
\hline
\end{tabular}

WHR, waist hip rate; Ref, reference; $\mathrm{OR}$, odds ratio; $\mathrm{Cl}$, confidence interval; $\mathrm{SD}$, standard deviation

Model I adjusted for age, sex, SBP, DBP, HR and BMI

Model II adjusted for age, sex, SBP, DBP, HR, BMI, smoking status, ALT, HDL-C, eGFR, DM, CHD, history of stroke and antihypertensive medications

had a higher prevalence of increased AS (adjusted-OR: 1.52, 95\% CI 1.28-1.81, $P<0.001$; Table 3). Compared to $\mathrm{WHR} \leq 0.87$, there were a higher prevalence of increased AS for the participants in the second and highest tertile of WHR (adjusted-OR: 1.36, 95\% CI 1.14-1.64, $P<0.001$; adjusted-OR: $1.81,95 \%$ CI 1.47-2.22, $P<0.001$, respectively; $P$ for trend $<0.001$; Table 3 ).

Stratified and interaction analyses indicated that the positive relationship between WHR levels and the prevalence of increased AS was still stable in the tertiles of age, SBP or DBP stratification no matter what using the WHR levels as continuous variable or the lower tertile of BMI as reference variable (all $P$-value for interaction respectively were greater than 0.05 in the main confounder model, Additional file 1: Table 7, 8 and 9). Likewise, subgroup analyses also indicated that the positive relationship between central obesity and the prevalence of increased AS were still stable in the tertiles of age, SBP or DBP stratification compared to those without central obesity (all $P$-value for interaction respectively were greater than 0.05 in the main confounder model, Additional file 1: Table 7, 8 and 9).

Relationship between the BMI levels and baPWV levels Linear regression models indicated that BMI levels were negatively associated with baPWV levels (adjusted- $\beta$ per 
$S D$ increase: $-0.57,95 \% C I-0.68$ to $-0.46, P<0.001$; Table 4). Compared to control group, patients with overweight and general obesity had lower baPWV levels (adjusted- $\beta$ : $-0.58,95 \% C I-0.79$ to $-0.37, P<0.001 ; \beta$ : $-1.20,95 \% C I-1.67$ to $-0.72, P<0.001$, respectively; $P$ for trend $<0.001$; Table 4). Compared to $B M I \leq 21.68 \mathrm{~kg} /$ $\mathrm{m}^{\wedge}$, there were lower baPWV levels in the second and highest tertile of BMI (adjusted- $\beta$ : $-0.61,95 \% C I-0.83$ to $-0.40, P<0.001 ; \beta$ : $-1.06,95 \% C I-1.31$ to -0.81 , $P<0.001$, respectively; $P$ for trend $<0.001$; Table 4 ). The smooth curve fitting further confirmed this reverse relationship between BMI levels and baPWV levels (Fig. 1).

To explore whether the negative correlation between BMI tertiles and baPWV levels were still stable in different subgroups, we conducted stratified and interaction analyses. There were not significant interactions in any of the following subgroups, including age tertiles, sex (male vs. female), SBP (<140 vs. $\geq 140 \mathrm{mmHg}), \mathrm{DBP}(<90$ vs. $\geq 90 \mathrm{mmHg}$ ), central obesity (no vs. yes), DM (no vs. yes), eGFR ( $\geq 60 \mathrm{vs} .<60 \mathrm{ml} / \mathrm{min} / 1.73 \mathrm{~m}^{\wedge}$ ) and antihypertensive medication (no vs. yes) (all $P$-value for interaction respectively were greater than 0.05 ; Additional file 1: Table 10).

\section{Relationship between the WHR levels and baPWV levels}

Linear regression models indicated that WHR levels were positively associated with baPWV levels (adjusted- $\beta$ per $S D$ increase: 4.46, 95\% CI 3.04-5.88, $P<0.001$; Table 5). Compared to subjects without central obesity, those with central obesity had greater baPWV levels (adjusted- $\beta$ : 0.54, 95\% CI 0.33-0.74, $P<0.001$; Table 5). Compared to $\mathrm{WHR} \leq 0.87$, there were greater baPWV levels in

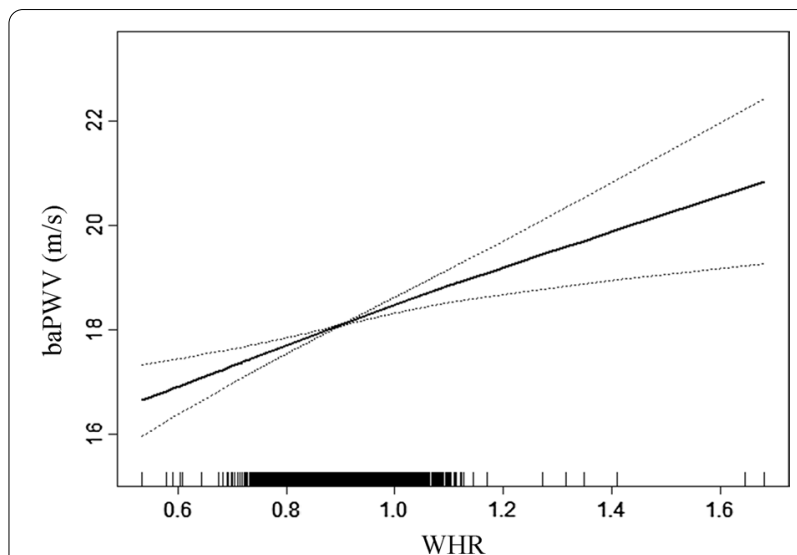

Fig. 1 Smooth curve of correlation between BMl levels and baPWV levels. BMI, Body mass index; baPWV, brachial-ankle pulse wave velocity. Smooth curve adjusted for age, sex, SBP, DBP, HR, WHR, eGFR, $\mathrm{DM}, \mathrm{CHD}$, history of stroke and antihypertensive medications.

the second and highest tertile of BMI (adjusted- $\beta$ : 0.49, 95\% CI 0.28-0.70, $P<0.001 ; \beta: 0.80,95 \%$ CI $0.56-1.04$, $P<0.001$, respectively; $P$ for trend $<0.001$; Table 5 ). The smooth curve fitting further confirmed this positive relationship between WHR levels and baPWV levels (Fig. 2).

To explore whether the positive correlation between WHR levels and baPWV levels were still stable in different subgroups, we also conducted stratified and interaction analyses. There were not significant interactions in any of the following subgroups, including age tertiles, sex (male vs. female), SBP (<140 vs. $\geq 140 \mathrm{mmHg}$ ), DBP ( $<90$ vs. $\geq 90 \mathrm{mmHg}$ ), smoking habit (no vs. yes), DM (no vs. yes), HDL ( $\geq 1.0$ vs. $<1.0 \mathrm{mmol} / \mathrm{L})$, eGFR ( $\geq 60$

Table 4 Relationship between BMI levels and baPWV levels in different models

\begin{tabular}{|c|c|c|c|c|c|c|}
\hline \multirow[t]{2}{*}{ Variables } & \multicolumn{2}{|l|}{ Crude Model } & \multicolumn{2}{|l|}{ Model I } & \multicolumn{2}{|l|}{ Model II } \\
\hline & $\beta(95 \% \mathrm{Cl})$ & P-value & $\beta(95 \% \mathrm{Cl})$ & $P$-value & $\beta(95 \% \mathrm{Cl})$ & $P$-value \\
\hline \multicolumn{7}{|l|}{$\mathrm{BMI}\left(\mathrm{kg} / \mathrm{m} \wedge^{2}\right)$} \\
\hline Per SD increase & $-0.72(-0.82,-0.61)$ & $<0.001$ & $-0.52(-0.63,-0.42)$ & $<0.001$ & $-0.57(-0.68,-0.46)$ & $<0.001$ \\
\hline \multicolumn{7}{|l|}{ BMI category $\left(\mathrm{kg} / \mathrm{m}^{\wedge} \wedge^{2}\right)$} \\
\hline Control $(<25)$ & Ref & & Ref & & Ref & \\
\hline Overweight $(\geq 25,<30)$ & $-1.02(-1.26,-0.78)$ & $<0.001$ & $-0.53(-0.74,-0.33)$ & $<0.001$ & $-0.58(-0.79,-0.37)$ & $<0.001$ \\
\hline General obesity $(\geq 30)$ & $-1.67(-2.26,-1.08)$ & $<0.001$ & $-1.14(-1.61,-0.66)$ & $<0.001$ & $-1.20(-1.67,-0.72)$ & $<0.001$ \\
\hline$P$-value for trend & $<0.001$ & & $<0.001$ & & $<0.001$ & \\
\hline \multicolumn{7}{|l|}{ BMI tertiles $\left(\mathrm{kg} / \mathrm{m}^{\wedge}{ }^{2}\right)$} \\
\hline T1 $[13.83,21.68]$ & Ref & & Ref & & Ref & \\
\hline T2 $[21.68,24.69]$ & $-1.06(-1.31,-0.80)$ & $<0.001$ & $-0.55(-0.77,-0.33)$ & $<0.001$ & $-0.61(-0.83,-0.40)$ & $<0.001$ \\
\hline T3 $[24.69,46.43]$ & $-1.62(-1.88,-1.37)$ & $<0.001$ & $-0.96(-1.21,-0.71)$ & $<0.001$ & $-1.06(-1.31,-0.81)$ & $<0.001$ \\
\hline$P$-value for trend & $<0.001$ & & $<0.001$ & & $<0.001$ & \\
\hline
\end{tabular}

BMI, body mass index; baPWV, brachial-ankle pulse wave velocity; Ref, reference; $\beta$, effect size; $\mathrm{Cl}$, confidence interval; $S D$, standard deviation Model I adjusted for age, sex, SBP, DBP, HR and WHR

Model II adjusted for age, sex, SBP, DBP, HR, WHR, eGFR, DM, CHD, history of stroke and antihypertensive medications 
Table 5 Relationship between WHR levels and baPWV levels in different models

\begin{tabular}{|c|c|c|c|c|c|c|}
\hline \multirow[t]{2}{*}{ Variables } & \multicolumn{2}{|l|}{ Crude Model } & \multicolumn{2}{|l|}{ Model I } & \multicolumn{2}{|l|}{ Model II } \\
\hline & $\beta(95 \% \mathrm{Cl})$ & P-value & $\beta(95 \% \mathrm{Cl})$ & P-value & $\beta(95 \% \mathrm{Cl})$ & P-value \\
\hline \multicolumn{7}{|l|}{ WHR } \\
\hline Per SD increase & $1.72(0.21,3.22)$ & 0.026 & $5.04(3.63,6.45)$ & $<0.001$ & $4.46(3.04,5.88)$ & $<0.001$ \\
\hline \multicolumn{7}{|l|}{ Central obesity } \\
\hline No & Ref & & Ref & & Ref & \\
\hline Yes & $0.22(-0.00,0.44)$ & 0.052 & $0.62(0.42,0.82)$ & $<0.001$ & $0.54(0.33,0.74)$ & $<0.001$ \\
\hline \multicolumn{7}{|l|}{ WHR tertiles } \\
\hline T1 $[0.53,0.87]$ & Ref & & Ref & & Ref & \\
\hline T2 $[0.87,0.93]$ & $-0.00(-0.26,0.26)$ & 0.995 & $0.54(0.32,0.75)$ & $<0.001$ & $0.49(0.28,0.70)$ & $<0.001$ \\
\hline T3 $[0.93,1.65]$ & $0.27(0.01,0.53)$ & 0.041 & $0.90(0.66,1.14)$ & $<0.001$ & $0.80(0.56,1.04)$ & $<0.001$ \\
\hline$P$-value for trend & $<0.001$ & & $<0.001$ & & $<0.001$ & \\
\hline
\end{tabular}

WHR, waist hip rate; baPWV, brachial-ankle pulse wave velocity; Ref, reference; $\beta$, effect size; $C l$, confidence interval; SD, standard deviation

Model I adjusted for age, sex, SBP, DBP, HR and BMI

Model II adjusted for age, sex, SBP, DBP, HR, WHR, smoking and drinking status, homocysteine, ALT, eGFR, HDL-C, DM, CHD, history of stroke and antihypertensive medications

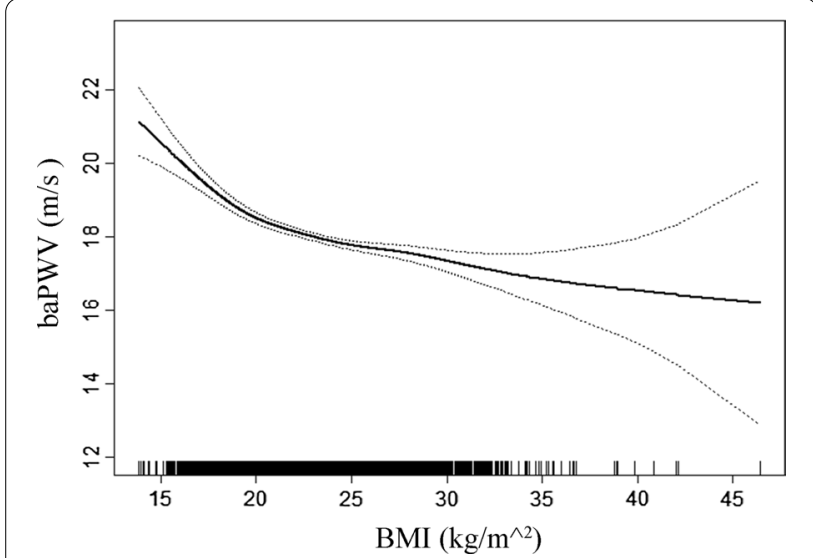

Fig. 2 Smooth curve of correlation between WHR levels and baPWV levels. WHR, waist hip rate; baPWV, brachial-ankle pulse wave velocity. Smooth curve adjusted for age, sex, SBP, DBP, HR, WHR, smoking and drinking status, homocysteine, ALT, eGFR, HDL-C, DM, CHD, history of stroke and antihypertensive medications

vs. $\left.<60 \mathrm{ml} / \mathrm{min} / 1.73 \mathrm{~m}^{\wedge 2}\right)$ and antihypertensive medication (no vs. yes) (all $P$-value for interaction respectively were greater than 0.05; Additional file 4: Fig. 3).

\section{Discussion}

In the present study, we showed an interesting finding that there was an inverse relationship of BMI levels with increased AS or baPWV, whereas WHR levels and central obesity were positively associated with increased AS or baPWV levels in Chinese rural adults with hypertension.

PWV was increased in hypertensive patients, and the degree of PWV increase was strongly linked to age and BP [11-13, 32, 40, 41]. In our study, nearly half (44.01\%) of the hypertensive participants had increased AS (baPWV $\geq 18 \mathrm{~m} / \mathrm{s}$ ) and baPWV levels were also positively associated with age, SBP and DBP (Additional file 1: Table 1 and 3). From Table 1 follows that with increasing BMI, not only age decreased (6.78 years between the lowest and highest tertile of BMI levels), but also SBP significantly decreased $(1.68 \mathrm{mmHg}$ between the lowest and highest tertile of BMI levels). Even more important, DBP increased $(4.52 \mathrm{mmHg}$ between the lowest and highest tertile of BMI). On the contrary, concerning the comparison of the two WHR groups, there are differences in terms of SBP or DBP, and that age decreased only 0.82 year, probably not significantly. In consideration of that age and BP were closely associated with PWV levels [11-13, 32, 40, 41], stratified and interaction analyses were conducted to explore the effects of age, SBP, and DBP on the association between BMI or WHR levels and the prevalence of increased AS or baPWV (Additional file 1: Table 4-10 and Additional file 1: Fig. 3). There were no interactions in terms of age and BP on the relationship between BMI or WHR levels and the prevalence of increased AS or baPWV levels.

Hypertension is often accompanied with obesity [3]. In our study, we found that the prevalence of overweight, general obesity, central obesity and were $26.88 \%, 3.39 \%$ and $63.85 \%$, respectively. In our analysis, only $5.12 \%$ of the 3,224 patients with central obesity were general obesity according to BMI categories, but $96.49 \%$ of the 171 patients with general obesity were central obesity (Table 1 and Additional file 1: Table 2). In other words, the relative weight of general obesity in the group of elevated WHR is very small. Inversely, the relative weight of elevated WHR in the group of 
general obesity is very high. Considering the fact that the proportion of patients with general obesity is very small $(3.39 \%)$ compared to the high proportion of subjects with central obesity (63.85\%), which might explain why the correlation of BMI and WHR levels with the prevalence of increased AS based on baPWV were not coincident.

Similar to our results, Huang et al. [29] and Liu et al. [18] found that there was a negative relationship between the BMI levels and baPWV levels among male hypertension participants. However, previous studies also showed that the BMI levels were positively correlated with PWV levels in Grade I essential hypertension [15] or obese and non-obese hypertensive patients [17]. On the one hand, different origins of the study participants might contribute to this discrepancy. The Liu et al. [18] study was based on 699 male hypertensive patients who were hospitalization or had other complications. The Samir et al. [15] study was based on 114 civil servants with Grade I essential hypertension. The Huang et al. [29] study enrolled 101, 510 participants, a coal occupation group in laborintensive enterprise, from 11 hospitals in the Kailuan community, which was most similar to our rural hypertensive patient populations. On the other hand, BMI is not able to differentiate fat-free muscle from fat mass. Furthermore, BMI cannot account for sex and racial differences in fat content, and it is not able to differentiate the distribution of visceral and subcutaneous fat [34]. The phenomenon of the obesity paradox may be related to genetics, cardiorespiratory fitness, beneficial adipose tissue and weaker sympathetic activation [34, 42, 43].

WHR, which is highly correlated with both increased visceral fat and low gluteal muscle mass [43-45], appears to be a more reliable prognosticator of CVDs than BMI $[35,36]$. Previous studies found that a high WHR was strongly associated with increased AS based on PWV $[19,25,27]$. The Whitehall II study, a prospective study of 10308 civil servants, showed that standardized effects of central adiposity on aortic PWV increase was obvious and previous adiposity was associated with aortic stiffening independent of change in adiposity, glycaemia, and lipid levels across PWV assessments [25]. We also demonstrated that WHR levels and central obesity were positively associated with increased AS or baPWV levels in Chinese rural adults with hypertension. Alexandre et al. [19] suggested that regional anthropometric indices including WHR were more closely correlated with PWV levels than BMI in hypertensive patients. Bouchi et al. [46], in a cross-sectional study with patients with diabetes and non-obese (normal BMI), noticed that increased visceral fat seems to be associated with increased AS based on baPWV. The lack of consistency between WHR and BMI may reflect that these measures identify different characteristics of obesity (central obesity in the case of WHR vs. subcutaneous/total fat in the case of BMI).

It is worth noting that eGFR was negatively associated with AS and baPWV in our study (Additional file 1: Table 1 and 3). Previous studies also showed that eGFR levels were reversely independently associated with cfPWV levels in patients with systemic lupus erythematosusa with a widely normally ranged eGFR levels (47). However, we cannot draw any causal relationship between eGFR levels and AS based on PWV in consideration of the cross-sectional analysis. Additionally, longitudinal studies indicated higher cfPWV levels were independently associated with steeper decline in eGFR levels and incident CKD in population more than 55 years old (48) and patients with type I diabetes (49). Higher cfPWV levels were also independently associated with the risk increased of progressive chronic kidney disease (CKD) in individuals with type II diabetes, and regression of PWV in the 3-year follow-up was associated with a lower risk of progressive CKD (50). With moderate progression of renal dysfunction and under well-controlled blood pressure during the 10-year follow-up period, peripheral AS based on baPWV but not central AS based on cfPWV was possibly one of the strongest predictors of CVD in patients with CKD stages III-V (51). These results suggest that AS based on PWV could be considered as a target for delaying decline in kidney function.

This study has several limitations. Firstly, this was a study of Chinese rural hypertensive patients and thus, the generalizability of the findings to other population remains to be determined. Secondly, the nutrional status such as the body shape might influence the measurement of baPWV causing information bias. Moreover, we cannot draw any causal relationship between the central obesity and AS based on baPWV considering the crosssectional analysis. In the future, additional large-scale longitudinal studies are required to determine if weight loss programs reverse AS and reduce the risk of cardiovascular events.

\section{Conclusion}

In conclusion, there was an inverse relationship between BMI levels and increased AS or baPWV, whereas WHR levels and central obesity were positively associated with increased AS or baPWV in Chinese rural adults with hypertension.

\footnotetext{
Abbreviations

CVDs: Cardiovascular diseases; AS: Arterial stiffness; cfPWV: Carotid-femoral pulse wave velocity; baPWV: Brachial-ankle pulse wave velocity; BMI: Body mass index; WHR: Waist hip rate; CHD: Coronary heart disease; SBP: Systolic blood pressure; DBP: Diastolic blood pressure; BP: Blood pressure; DM: Diabetes mellitus; FBG: Fasting blood glucose; TC: Total cholesterol; TG: Total
} 
triglyceride; HDL-C: High-density lipoprotein cholesterol; LDL-C: Low-density lipoprotein cholesterol; BUN: Blood urea nitrogen; eGFR: Estimated glomerular filtration rate; AST: Aspartate aminotransferase; ALT: Alanine aminotransferase; CKD-EPI: Chronic kidney disease epidemiology collaboration; CKD: Chronic kidney disease; SD: Standard deviation; OR: Odds ratio; Cl: Confidence interval.

\section{Supplementary Information}

The online version contains supplementary material available at https://doi. org/10.1186/s12872-021-02390-y.

Additional file 1. Supplementary Table 1. Association of covariates with the prevalence of increased arterial stiffness and baPWV levels. Supplementary Table 2. Relationship between BMI levels and WHR levels or central obesity. Supplementary Table 3. Clinical characteristics of participants grouped by baPWV quartiles. Supplementary Table 4. Relationship between BMI levels and the prevalence of increased arterial stiffness stratified by age. Supplementary Table 5. Relationship between BMI levels and the prevalence of increased arterial stiffness stratified by SBP. Supplementary Table 6 . Relationship between BMI levels and the prevalence of increased arterial stiffness stratified by DBP. Supplementary Table 7. Relationship between WHR levels and the prevalence of increased arterial stiffness stratified by age. Supplementary Table $\mathbf{8}$. Relationship between WHR levels and the prevalence of increased arterial stiffness stratified by SBP. Supplementary Table $\mathbf{9}$. Relationship between WHR levels and the prevalence of increased arterial stiffness stratified by DBP. Supplementary Table 10. Effect size of BMI tertiles on baPWV levels in prespecified and exploratory subgroups.

Additional file 2: Fig. 1 The data flow chart of participants in our analysis. baPWV, brachial-ankle pulse wave velocity; BMI, body mass index; ABI, ankle brachial index; AF, atrial fibrillation; WHR, waist hip rate.

Additional file 3: Fig. 2 The correlation between BMI levels and WHR levels.

Additional file 4: Fig. 3 Effect size of central obesity on baPWV levels in prespecified and exploratory subgroups. SBP, systolic blood pressure; DBP, diastolic blood pressure; DM, diabetes mellitus; HDL-C, high-density lipoprotein cholesterol; eGFR, estimated glomerular filtration rate $\beta$, effect size; $\mathrm{Cl}$, confidence interval. Each stratification adjusted for age, sex, SBP, DBP, $\mathrm{HR}$, WHR, smoking and drinking status, homocysteine, ALT, eGFR, HDL-C, $\mathrm{DM}, \mathrm{CHD}$, history of stroke and antihypertensive medications except the subgroup variable.

\section{Acknowledgements}

Not applicable.

\section{Authors' contributions}

$\mathrm{FH}$ and $\mathrm{RHY}$ participated in literature searches, data analysis, and data interpretation. FH wrote the manuscript. RHY extracted and collected data. $\mathrm{FYH}$, $J$ L, WZ, TW, LJZ, and XH conceived of the study and participated in its design and coordination. HHB and XSC participated in the study design and provided critical revisions. All authors read and approved the final manuscript.

\section{Funding}

This work was supported by the National Natural Science Foundation of China (81760049, 82160070), the Jiangxi Science and Technology Innovation Platform Project (20165BCD41005), the National Key R\&D Program of China (2018YFC1312902), the Key Project of Education Department of Jiangxi Province (GJJ170013), the Natural Science Foundation of Jiangxi Province of China (20192BAB205033), the Fund project of the Second Affiliated Hospital of Nanchang University (2016YNQN12034, 2019YNLZ12010).

\section{Availability of data and materials}

The datasets used and analysed during the current study available from the corresponding author on reasonable request.

\section{Declarations}

\section{Ethics approval and consent to participate}

The study was conducted in accordance with the Declaration of Helsinki, and the protocol was approved by the Ethics Committee of Institute of Biomedicine, Anhui Medical University. Signed informed consents were obtained from all individuals.

\section{Consent for publication}

Not applicable.

\section{Competing interests}

The authors declare that they have no competing interests.

\section{Author details}

${ }^{1}$ Department of Cardiovascular Medicine, The Second Affiliated Hospital of Nanchang University, No. 1 Minde Road, Nanchang 330006, Jiangxi, China. ${ }^{2}$ Jiangxi Provincial Cardiovascular Disease Clinical Medical Research Center, Nanchang, Jiangxi, China. ${ }^{3}$ Department of General Practice Medicine, Zhentou Town Health Center, Wuyuan, Jiangxi, China. ${ }^{4}$ The College of Pharmacy, Nanchang University, Nanchang, Jiangxi, China. ${ }^{5}$ Center for Prevention and Treatment of Cardiovascular Diseases, The Second Affiliated Hospital of Nanchang University, Nanchang, Jiangxi, China.

Received: 29 December 2020 Accepted: 12 November 2021

Published online: 01 December 2021

\section{References}

1. Mensah GA, Roth GA, Fuster V. The Global Burden of Cardiovascular Diseases and Risk Factors: 2020 and Beyond. J Am Coll Cardiol. 2019:74(20):2529-32.

2. Blüher M. Obesity: global epidemiology and pathogenesis. Nat Rev Endocrinol. 2019:15(5):288-98.

3. Liu J. Highlights of the 2018 Chinese hypertension guidelines. Clin Hypertens. 2020;26:8.

4. Whelton PK, Carey RM, Aronow WS, Casey DJ, Collins KJ, Dennison HC, et al. 2017 ACC/AHA/AAPA/ABC/ACPM/AGS/APhA/ASH/ASPC/NMA/

5. PCNA Guideline for the Prevention. Detection, evaluation, and management of high blood pressure in adults: a report of the american college of cardiology/American heart association task force on clinical practice guidelines. Hypertension. 2018;71(6):e13-115.

6. Mitchell GF, Hwang SJ, Vasan RS, Larson MG, Pencina MJ, Hamburg NM, et al. Arterial stiffness and cardiovascular events: the Framingham Heart Study. Circulation. 2010;121(4):505-11.

7. Laurent S, Boutouyrie P, Asmar R, Gautier I, Laloux B, Guize L, et al. Aortic stiffness is an independent predictor of all-cause and cardiovascular mortality in hypertensive patients. Hypertension. 2001;37(5):1236-44.

8. Munakata M. Brachial-ankle pulse wave velocity in the measurement of arterial stiffness: recent evidence and clinical applications. Curr Hypertens Rev. 2014;10(1):49-57.

9. Yu WC, Chuang SY, Lin YP, Chen CH. Brachial-ankle vs carotid-femoral pulse wave velocity as a determinant of cardiovascular structure and function. J Hum Hypertens. 2008;22(1):24-31.

10. Yamashina A, Tomiyama H, Takeda K, Tsuda H, Arai T, Hirose K, et al. Validity, reproducibility, and clinical significance of noninvasive brachial-ankle pulse wave velocity measurement. Hypertens Res. 2002;25(3):359-64.

11. Kubozono T, Miyata M, Kawasoe S, Ojima S, Yoshifuku S, Miyahara H, et al. High pulse wave velocity has a strong impact on early carotid atherosclerosis in a Japanese general male population. Circ J. 2017;81(3):310-5.

12. Qu G, Zhang Z, Zhu H. Association between blood pressure control and arterial stiffness in middle-aged and elderly Chinese patients with hypertension. Med Sci Monit. 2021;27:e931414.

13. Wu S, Jin C, Li S, Zheng X, Zhang X, Cui L, et al. Aging, arterial stiffness, and blood pressure association in Chinese adults. Hypertension. 2019;73(4):893-9.

14. Lu Y, Pechlaner R, Cai J, Yuan H, Huang Z, Yang G, et al. Trajectories of agerelated arterial stiffness in Chinese men and women. J Am Coll Cardiol. 2020;75(8):870-80. 
15. Tan J, Hua Q, Xing X, Wen J, Liu R, Yang Z. Impact of the metalloproteinase-9/tissue inhibitor of metalloproteinase-1 system on large arterial stiffness in patients with essential hypertension. Hypertens Res. 2007;30(10):959-63

16. Mallat S, Jurjus A, Bou Assi T, Jurjus A, Shams A, El Sayegh S, et al. Markers of arterial stiffness in a sample of Lebanese subjects with Grade I essential hypertension. SAGE Open Med. 2017;5:2050312117712886.

17. Park S, Kim JB, Shim CY, Ko YG, Choi D, Jang Y, et al. The influence of serum aldosterone and the aldosterone-renin ratio on pulse wave velocity in hypertensive patients. J Hypertens. 2007;25(6):1279-83.

18. Toto-Moukouo JJ, Achimastos A, Asmar RG, Hugues CJ, Safar ME. Pulse wave velocity in patients with obesity and hypertension. Am Heart J. 1986;112(1):136-40.

19. Liu Y, Qi LT, Ma W, Yang Y, Meng L, Zhang BW, et al. Correlation between anthropometric parameters and arteriosclerosis biomarker in the middle-aged and the elderly. Beijing Da Xue Xue Bao Yi Xue Ban. 2014;46(3):455-9.

20. Vallée A, Olié V, Lelong HÉL, Kretz S, Safar ME, Blacher J. Relationship between BMI and aortic stiffness: influence of anthropometric indices in hypertensive men and women. J Hypertens. 2020;38(2):249-56.

21. Zemtsovskaja G, Abina J, Meigas K, Pilt K, Viigimaa M. Pulse wave velocity and its gender-related associations with cardiovascular risk factors in a high cardiovascular risk population. Arch Med Sci Atheroscler Dis. 2018:3:e99-105.

22. Czernichow $S$, Bertrais S, Oppert JM, Galan P, Blacher J, Ducimetière $P$, et al. Body composition and fat repartition in relation to structure and function of large arteries in middle-aged adults (the SU.VI.MAX study). Int J Obes. 2005;29(7):826-32.

23. Kolade OO, O'Moore-Sullivan TM, Stowasser M, Coombes JS, Fassett RG, Marwick TH, et al. Arterial stiffness, central blood pressure and body size in health and disease. Int J Obes. 2012;36(1):93-9.

24. Haraguchi N, Koyama T, Kuriyama N, Ozaki E, Matsui D, Watanabe I, et al. Assessment of anthropometric indices other than BMI to evaluate arterial stiffness. Hypertens Res. 2019;42(10):1599-605.

25. Choi HS, Cho YH, Lee SY, Park EJ, Kim YJ, Lee JG, et al. Association between new anthropometric parameters and arterial stiffness based on brachialankle pulse wave velocity. Diabetes Metab Syndr Obes. 2019;12:1727-33.

26. Brunner EJ, Shipley MJ, Ahmadi-Abhari S, Tabak AG, McEniery CM, Wilkinson IB, et al. Adiposity, obesity, and arterial aging: longitudinal study of aortic stiffness in the Whitehall II cohort. Hypertension. 2015;66(2):294-300.

27. Vianna CA, Horta BL, Gonzalez MC, França GVA, Gigante DP, Barros FL. Association of pulse wave velocity with body fat measures at $30 \mathrm{y}$ of age. Nutrition. 2019;61:38-42.

28. Wildman RP, Mackey RH, Bostom A, Thompson T, Sutton-Tyrrell K. Measures of obesity are associated with vascular stiffness in young and older adults. Hypertension. 2003;42(4):468-73.

29. Kulsum-Mecci N, Goss C, Kozel BA, Garbutt JM, Schechtman KB, Dharnidharka VR. Effects of obesity and hypertension on pulse wave velocity in children. J Clin Hypertens. 2017;19(3):221-6.

30. Huang J, Chen Z, Yuan J, Zhang C, Chen H, Wu W, et al. Association between body mass index (BMI) and brachial-ankle pulse wave velocity (baPWV) in males with hypertension: a community-based cross-section study in North China. Med Sci Monit. 2019;25:5241-57.

31. Gomez-Sanchez L, Garcia-Ortiz L, Patino-Alonso MC, Recio-Rodriguez J, Rigo F, Martí R, et al. Adiposity measures and arterial stiffness in primary care: the MARK prospective observational study. BMJ Open. 2017;7(9):e016422.

32. Yang $H$, Zhao J, Deng $X$, Tan I, Butlin M, Avolio A, et al. Pulse wave velocity is decreased with obesity in an elderly Chinese population. J Clin Hypertens. 2019;21(9):1379-85.

33. Lurbe E, Torro I, Garcia-Vicent C, Alvarez J, Fernández-Fornoso JA, Redon J. Blood pressure and obesity exert independent influences on pulse wave velocity in youth. Hypertension. 2012;60(2):550-5.

34. Dangardt F, Osika W, Volkmann R, Gan LM, Friberg P. Obese children show increased intimal wall thickness and decreased pulse wave velocity. Clin Physiol Funct Imaging. 2008;28(5):287-93.

35. Ahima RS, Lazar MA. Physiology. The health risk of obesity-better metrics imperative. Science. 2013;341:856-8.
36. Elsayed EF, Tighiouart H, Weiner DE, Griffith J, Salem D, Levey AS, et al. Waist-to-hip ratio and body mass index as risk factors for cardiovascular events in CKD. Am J Kidney Dis. 2008;52(1):49-57.

37. Streng KW, Voors AA, Hillege H, Anker SD, Cleland JG, Dickstein K, et al. Waist-to-hip ratio and mortality in heart failure. Eur J Heart Fail. 2018;20(9):1269-77

38. Li M, Zhan A, Huang X, Hu L, Zhou W, Wang T, et al. Positive association between triglyceride glucose index and arterial stiffness in hypertensive patients: the China H-type Hypertension Registry Study. Cardiovasc Diabetol. 2020;19(1):139.

39. Yin J, Li M, Yu L, Hu F, Yu Y, Hu L, et al. The relationship between the atherogenic index of plasma and arterial stiffness in essential hypertensive patients from China: a cross-sectional study. BMC Cardiovasc Disord. 2021:21(1):245

40. Liu Z, Yang H, Chen S, Cai J, Huang Z. The association between body mass index, waist circumference, waist-hip ratio and cognitive disorder in older adults. J Public Health. 2019;41(2):305-12.

41. Lovic D, Kallistratos MS, Poulimenos L, Skyrlas A, Stojanov V, Jakovljevic $B$, et al. Pulse wave velocity: retrospective analysis in a Balkan normotensive and hypertensive population: a study of 9923 patients. Angiology. 2018;69(1):59-64

42. Diaz A, Tringler M, Wray S, Ramirez AJ, Cabrera Fischer El. The effects of age on pulse wave velocity in untreated hypertension. J Clin Hypertens (Greenwich). 2018:20(2):258-65.

43. Sharma A, Lavie CJ, Borer JS, Vallakati A, Goel S, Lopez-Jimenez F, et al. Meta-analysis of the relation of body mass index to all-cause and cardiovascular mortality and hospitalization in patients with chronic heart failure. Am J Cardiol. 2015;115(10):1428-34.

44. Seo DC, Choe S, Torabi MR. Is waist circumference $\geq 102 / 88 \mathrm{~cm}$ better than body mass index $\geq 30$ to predict hypertension and diabetes development regardless of gender, age group, and race/ethnicity? Metaanalysis Prev Med. 2017;97:100-8.

45. Fox CS, Massaro JM, Hoffmann U, Pou KM, Maurovich-Horvat P, Liu CY, et al. Abdominal visceral and subcutaneous adipose tissue compartments: association with metabolic risk factors in the Framingham Heart Study. Circulation. 2007;116(1):39-48.

46. Schoen RE, Thaete FL, Sankey SS, Weissfeld JL, Kuller LH. Sagittal diameter in comparison with single slice CT as a predictor of total visceral adipose tissue volume. Int J Obes Relat Metab Disord. 1998;22(4):338-42.

47. Bouchi R, Minami I, Ohara N, Nakano Y, Nishitani R, Murakami M, et al. Impact of increased visceral adiposity with normal weight on the progression of arterial stiffness in Japanese patients with type 2 diabetes. BMJ Open Diabetes Res Care. 2015;3(1):e000081.

48. Stortz M, Triantafyllias K, Schwarting A, Weinmann-Menke J. Vascular stiffness: influencing factors on carotid-femoral pulse wave velocity in systemic lupus erythematosus. Clin Exp Rheumatol. 2020;38(1):74-81.

49. Sedaghat S, Mattace-Raso FU, Hoorn EJ, Uitterlinden AG, Hofman A, Ikram MA, et al. Arterial stiffness and decline in kidney function. Clin J Am Soc Nephrol. 2015;10(12):2190-7.

50. Tougaard NH, Theilade S, Winther SA, Tofte N, Ahluwalia TS, Hansen TW, et al. Carotid-femoral pulse wave velocity as a risk marker for development of complications in type 1 diabetes mellitus. J Am Heart Assoc. 2020;9(19):e017165.

51. Liu JJ, Liu S, Lee J, Gurung RL, Yiamunaa M, Ang K, et al. Aortic pulse wave velocity, central pulse pressure, augmentation index and chronic kidney disease progression in individuals with type II diabetes: a 3-year prospective study. BMC Nephrol. 2020;21(1):359.

52. Suzuki H, Inoue T, Dogi M, Kikuta T, Takenaka T, Okada H. Role of pulse wave velocity in patients with chronic kidney disease stages 3-5 on longterm follow-up. Pulse (Basel). 2014:2(1-4):1-10.

\section{Publisher's Note}

Springer Nature remains neutral with regard to jurisdictional claims in published maps and institutional affiliations. 